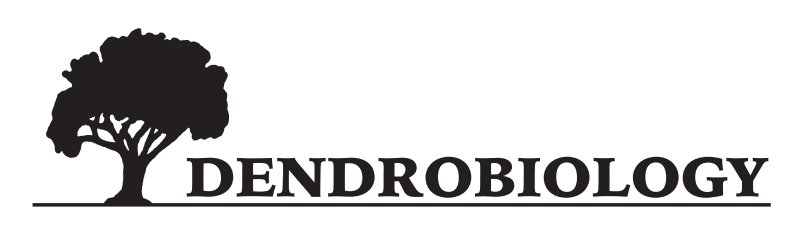

2016, vol. 75, 123-129

http://dx.doi.org/10.12657/denbio.075.012

\author{
Huaizhi Mu, Lin Lin, Qiuyan Zhang, Xiaojie Tang, Xiao Zhang, \\ Guangyou Cheng*
}

\title{
Growth, proline content and proline-associated gene expression of autotetraploid Betula platyphylla responding to $\mathrm{NaHCO}_{3}$ stress
}

Received: 31 May 2015; Accepted: 25 November 2015

\begin{abstract}
Plant breeders have focused much attention on polyploid trees because of their resistance for forestry. To evaluate the impact of intraspecies genome duplication on $\mathrm{NaHCO}_{3}$ stress, a series of Betula platyphylla autotetraploids and diploids were generated from the same family. The growth, proline content and proline-associated gene expression of these autotetraploid individuals were compared with those diploid trees. Autotetraploids were superior in injury index and relative growth of height and base diameter compared to diploids. The proline content was higher in autotetraploid individuals compared to diploids. Gene expression data revealed autotetraploids were generally higher expression in BpP5CS1, BpP5CS2, BpP5CR1, BpP5CR2, BpP5CR3 and BpOAT and were lower expression in BpProDH and BpP5CDH compared to diploid trees. These results shed light on resistance variation in birch autotetraploidization and polyploidy breeding as a new approach for genetic improvement of birch trees.
\end{abstract}

Keywords: birch, polyploid, stress response

Addresses: H. Z. Mu, X. J. Tang, X. Zhang, G. Y. Cheng*, Key Laboratory for Forestry and Ecological Environment of Jilin Province, Forestry College, Beihua University, 132013, Jilin, China, e-mail: huaizhimu@126.com; cgy6868@sina.com

L. Lin, Research Center of Cold Temperate Forestry, Chinese Academy of Forestry, 150086, Harbin, China Harbin Research Institute of Forestry Machinery, China State Forestry Administration, 150086, Harbin, China

Q. Y. Zhang, Jiaohe Forestry Experimental Area Administration, Jilin Province, 132517, Jiaohe, China *Corresponding author

\section{Introduction}

Salt and alkali are common environmental stresses which have great impact on plant productivity. Saline-alkali soli is widely distributed throughout the world and increases year by year (Ahmed, 1991). How to use saline-alkali soli to develop agriculture and forestry is an urgent issue. Saline-alkali soli improvement is increasingly constrained by freshwater resource. Consequently, the breeding of new varieties having strong salt and alkali tolerance is an effective method to utilize saline-alkali soli. To breed new varieties of salt and alkali tolerant using the traditional method was simple, but the work 
moved slowly, has not yet made true salt and alkali tolerant varieties. With the development of breeding technology, researchers want to use new technology for breeding new varieties having salt and alkali tolerance for agriculture and forestry production in salinization area.

Polyploidization events occurred frequently during plant evolution and most polyploids occurred in harsh environment, means polyploid plants had stronger ability to adapt to adverse natural conditions compared to diploids (Deng et al., 2012). In forestry, polyploids in Populus and Robinia have been shown to exhibit higher resistance to salt, drought and disease stress compared to their diploid counterparts, has been applied in production (Johnsson, 1953; Niwa \& Sasaki, 2003; Meng et al., 2008; Liu et al., 2012).

The members of the genus Betula form a particularly significant group of broad-leaved trees in Eurasia and North America. Certain birch species, such as B. platyphylla, B. pendula, B. pubescens and B. papyrifera, are valuable sources of wood, and great importance is attached to breeding work aimed at their economic improvement (Eriksson \& Jonsson, 1986). A natural European birch (B. verrucosa) triploid, discovered by Löve (1944), displayed more resistant to birch rust (Melampsoridium betulinum) (Eifler, 1960). Pentaploid and hexaploid birch (B. papyrifera) were more tolerant of water deficit than their diploid relatives (Li et al., 1996). No other polyploid cultivars in Betula had been employed in production although there were some attempts (Koski \& Rousi, 2005). In 2012, we compared differences between transcriptomes of diploid and autotetraploid B. platyphylla plants using Illumina paired-end sequencing technology. The results revealed that biosynthesis and degradation of proline differed significantly between autotetraploids and diploids (Mu et al., 2012). In response to this problem, autotetraploids and diploids generated from the same family were subjected to $\mathrm{NaHCO}_{3}$ stress. By investigating changes in growth, proline content and proline-associated gene expression attributable to genome duplication, our study deepens our understanding of resistance variation in birch autotetraploidization and polyploidy breeding as a new approach for genetic improvement of birch trees.

\section{Materials and Methods}

\section{Plant materials}

The experiment was initiated in August 2013. Seeds from the same family were soaked in $0.1 \%$ $(w / v)$ colchicine for $48 \mathrm{~h}$ in the dark. A number of seeds from the family were soaked in distilled water for the controls. The seeds were sown in a greenhouse after colchicine treatment, and the saplings were evaluated by ploidy measurement ( $\mathrm{Mu}$ et al., 2012). In 2014, 76 autotetraploid saplings and 70 diploid saplings were transplanted into plastic pots in the greenhouse. In July 2014, 35 individual clones of autotetraploid and diploids chosen at random were irrigated by 2 liters of $0.8 \%(w / v) \mathrm{NaHCO}_{3}$ solution every 3 days lasting for 12 days in total.

\section{Injury index and relative growth measurements}

Injury index was calculated after $\mathrm{NaHCO}_{3}$ stress and the calculation was as follows: a green leaf was marked 0; a leaf whose margin was slightly yellow, was marked 1; a leaf whose half was yellow, was marked 2; a leaf whose three-quarter was yellow, was marked 3; a sere leaf was marked 4. The injury index calculation was performed as described by Sun et al. (2002) as follows:

$$
\mathrm{I}=\sum(\mathrm{i} \times \mathrm{n}) / \mathrm{N}
$$

where I and $\mathrm{i}$ indicate the injury index of a sapling and a leaf, respectively, and $\mathrm{N}$ and $\mathrm{n}$ indicate the number of all leaves in a sapling and the leaves having corresponding injury index, respectively.

Height and base diameter of diploid and autotetraploid saplings were measured before $\mathrm{NaHCO}_{3}$ stress. After $\mathrm{NaHCO}_{3}$ irrigated, diploids and autotetraploids were managed in normal water and fertilizer in the greenhouse. The measurements of height and base diameter were undertaken again after the end of vegetation period during the first year. The relative growth of height and diameter was calculated as described by Li et al. (2013) as follows:

$$
R G=(A G-B G) / B G \times 100 \%
$$

where RG is the relative growth of height and diameter of a sapling, AG and BG indicate the height and base diameter of a sapling which is after the end of vegetation period during the first year and before $\mathrm{NaHCO}_{3}$ stress, respectively. Meanwhile, the relative growth of diploids and autotetraploids was calculated in non stress condition.

\section{Proline measurement}

The measurement of proline was carried out during the first summer. The $3^{\text {rd }}$ leaf from the apical meristem of each sapling was obtained at the $0^{\text {th }}$ day, $3^{\text {rd }}$ day, $6^{\text {th }}$ day, $9^{\text {th }}$ day and $12^{\text {th }}$ day in $\mathrm{NaHCO}_{3}$ irrigating respectively, and were immediately frozen in liquid nitrogen and stored at $-80^{\circ} \mathrm{C}$. The frozen leaves 
Growth, proline content and proline-associated gene expression of autotetraploid Betula platyphylla... 125

were divided into two groups: one group of frozen leaves was used for proline measurement; the other group of frozen leaves was used for proline-associated genes analysis.

Proline was extracted from the leaves with $3 \%$ $(w / v)$ sulfosalicylic acid at $100^{\circ} \mathrm{C}$ for $10 \mathrm{~min}$. After $2.5 \%(w / v)$ acidic ninhydrin coloration, the spectrum of proline in the extracts was scanned with a spectrophotometer (722, TP, China). Total proline quantification was performed as described by Zhang et al. (1990). All experiments were conducted with three technical replicates for each sample.

\section{Proline-associated genes analysis}

According to transcriptome data of diploid and autotetraploid B. platyphylla trees using Illumina paired-end sequencing technology (Mu et al., 2012), eight genes (BpP5CS1, BpP5CS2, BpP5CR1, BpP5CR2, BpP5CR3, BpOAT, BpProDH and BpP5CDH) involved in biosynthesis and degradation of proline were selected for real-time quantitative RT-PCR (q-PCR) analysis. Primers for these genes were designed manually (Table 1). RNA from each sample was isolated using a modified CTAB method (Zeng et al., 2007) and digested with DNase I (RNase free) to remove contaminating DNA. The first-strand cDNA was synthesized using ReverTra Ace qPCR RT Master Mix with gDNA Remover (Toyobo, Osaka, Japan) according to the manufacturer protocol. The cDNA was diluted tenfold and used as the template for q-PCR.

The q-PCR mixture comprised a $3 \mu \mathrm{L}$ template of the RT reaction mixture, $10 \mu \mathrm{L}$ of $2 \times$ SYBR Green Master Mix (Toyobo, Osaka, Japan) and $0.5 \mu \mathrm{L}$ of forward and reverse primer $(10 \mu \mathrm{mol} / \mathrm{L})$ brought to a final volume of $20 \mu \mathrm{L}$ with water. The reactions were performed in an MJ Opticon ${ }^{\mathrm{TM}-2}$ machine (Bio-Rad, Hercules, CA, USA) using the two-step method that was initiated for $30 \mathrm{~s}$ at $94^{\circ} \mathrm{C}$ followed by 44 cycles of $94^{\circ} \mathrm{C}$ for $12 \mathrm{~s}$, $55^{\circ} \mathrm{C}$ for $30 \mathrm{~s}, 72^{\circ} \mathrm{C}$ for $30 \mathrm{~s}$ and $78.5^{\circ} \mathrm{C}$ for $1 \mathrm{~s}$ for plate reading. A melting curve was performed from $55^{\circ} \mathrm{C}$ to $95^{\circ} \mathrm{C}$ to check the specificity of the amplified product. All experiments were conducted with three technical replicates for each sample. The expression was calculated by $2^{-\Delta \Delta C t}$ and normalized to values obtained from the 18S rRNA and $\alpha$-tubulin controls.

\section{Results}

\section{Injury index and relative growth}

The phenotypes of autotetraploid trees differed significantly from diploids after $\mathrm{NaHCO}_{3}$ irrigated. The mean injury index of autotetraploids was only 1.58 , which was $31.15 \%$ lower than that of diploids. The maximal injury index of the autotetraploid was only 2.02 , which was $11.79 \%$ lower than the mean injury index observed for the diploids (Fig. 1). In non stress condition, the relative growth of height and base diameter of autotetraploids were $34.80 \%$ and $2.80 \%$ lower than those of diploids, respectively(Fig. 2a). Nevertheless, the relative growth of height and base diameter of autotetraploids were $20.19 \%$ and $18.44 \%$ larger than those of diploids in $\mathrm{NaHCO}_{3}$ stress condition, respectively (Fig. 2b). These results indicate that the injury degree of autotetraploids was lower than that of diploids, and the relative increase in height and base diameter of au-

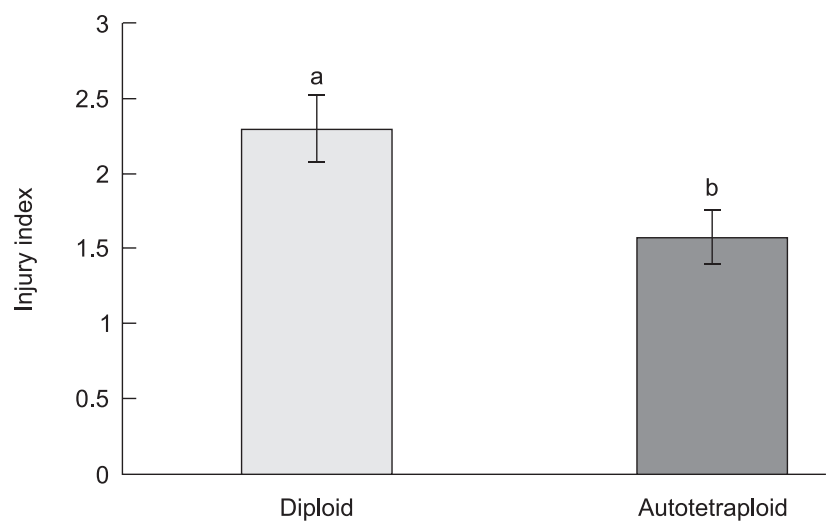

Fig. 1. Injury index of diploids and autotetraploids after $\mathrm{NaHCO}_{3}$ irrigated. Small letters at the top of error bars show results of Duncan test for each female parent separately, columns with different letters differ significantly $(P<0.05)$

Table 1. Primers used for real-time quantitative RT-PCR analysis.

\begin{tabular}{|c|c|c|}
\hline Gene & Forward primer $\left(5^{\prime}-3^{\prime}\right)$ & Reverse primer $\left(5^{\prime}-3^{\prime}\right)$ \\
\hline $18 S$ rRNA & ATCTTGGGTTGGGCAGATCG & CATTACTCCGATCCCGAAGG \\
\hline$\alpha$-Tublin & GCACTGGCCTCCAAGGAT & TGGGTCGCTCAATGTCAAGG \\
\hline BpP5CS1 & CATCTCGGACAGCAAAGT & GGTAACCCACAATAATGAAGTA \\
\hline BpP5CS2 & CATCTCGGACAGCAAAGT & CACTGGTAACCCACAATA \\
\hline BpP5CR1 & CTCCTCCGTCAGCCAAAG & ATGCCAAATACTCCATCAGC \\
\hline BpP5CR2 & TACGGGAAGGAGGCAACA & CAAACCAGACGACCCACTAA \\
\hline BpP5CR3 & CTCCTCCGTCAGCCAAAG & ATGCCAAATACTCCATCAGC \\
\hline ВрОАТ & TAGTTCGGGTATTGCTGC & ATGTTGGCTTGTGATTGG \\
\hline BpProDH & GGTTGAGCGGGTGAGTGA & GCGGGTTGGATGGAAGTGT \\
\hline $\mathrm{BPP} 5 \mathrm{CDH}$ & GCAGGATTCGGCACGAGGCAT & GCAGTTGCTCCTCAAGTAGATGCAG \\
\hline
\end{tabular}




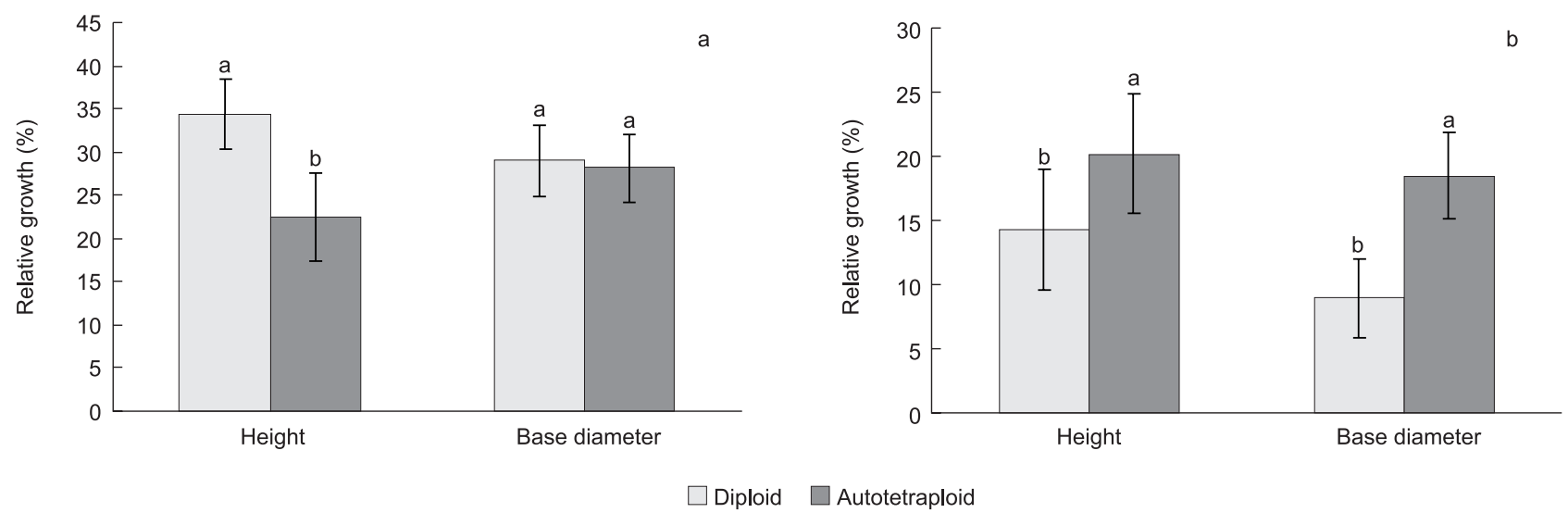

Fig. 2. Relative growth of height and base diameter of diploids and autotetraploids in non stress condition (a) and NaH$\mathrm{CO}_{3}$ stress condition (b), respectively

totetraploids were superior to those of diploids after $\mathrm{NaHCO}_{3}$ stress. Consequently, the autotetraploid trees were more tolerant to salt and alkali stress than diploids.

\section{Proline content}

The proline variation of diploids and autotetraploids was similar in $\mathrm{NaHCO}_{3}$ irrigating, which showed a downward trend after the first rise. Nevertheless, the proline content of autotetraploid saplings was always higher than that of diploids. The proline level of autotetraploids peaked in the $9^{\text {th }}$ day $\left(17.59 \mu \mathrm{g} \mathrm{g}^{-1}\right)$, and this was $114.51 \%$ greater than that of diploids. The proline level of diploids peaked in the $6^{\text {th }}$ day (mean proline content of $10.52 \mu \mathrm{g} \cdot \mathrm{g}^{-1}$ ), and this was $33.12 \%$ lower than that of autotetraploids (Fig. 3).

\section{Proline-associated gene expression}

In order to study the relationship between the proline content and proline-associated gene expression, we investigated the relative expression levels of eight genes involved in biosynthesis and degrada-

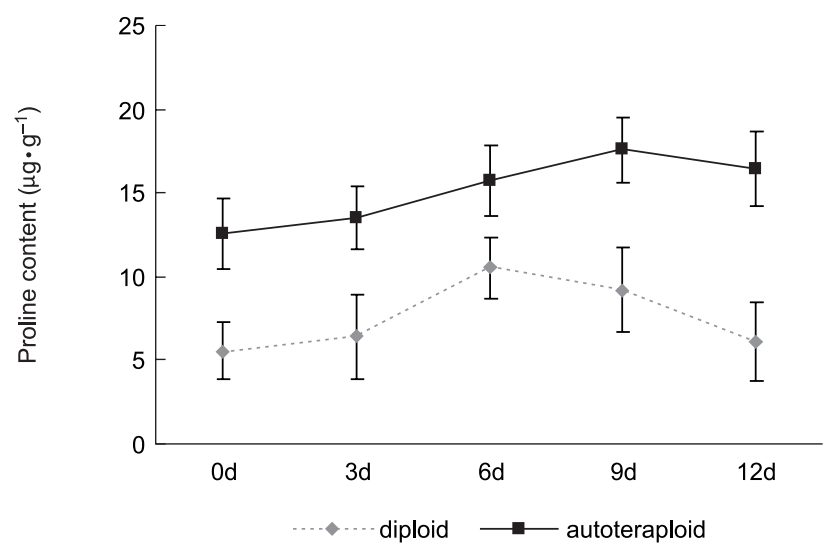

Fig. 3. Proline content of diploids and autotetraploids in $\mathrm{NaHCO}_{3}$ irrigating tion of proline in $\mathrm{NaHCO}_{3}$ irrigating. The expression of BpP5CS1 and BpP5CS2 in diploids and autotetraploids showed a downward trend after the first rise, and which of autotetraploids were always higher than those of diploids. BpP5CS1 and BpP5CS2 expression levels of autotetraploids peaked in the $9^{\text {th }}$ day, and there were $120.30 \%$ and $1549.64 \%$ greater than those of diploids, respectively. BpP5CS1 and BpP5CS2 expression levels of diploids peaked in the $6^{\text {th }}$ day, and there were $16.17 \%$ and $4.92 \%$ lower than those of autotetraploids, respectively (Fig. 4a-b). The expression of BpP5CR1, BpP5CR2 and BpP5CR3 in diploids and autotetraploids showed a downward trend after the first rise, and the expression of BpP5CR2 and Bp$P 5 C R 3$ of autotetraploids were always higher than those of diploids. BpP5CR1, BpP5CR2 and BpP5CR3 expression levels of autotetraploids peaked in the $9^{\text {th }}$ day, and there were $232.42 \%, 57.27 \%$ and $109.77 \%$ greater than those of diploids, respectively (Fig. 4ce). The expression of BpOAT in diploids and autotetraploids showed a rising trend, and which of autotetraploids was always higher than that of diploids. BpOAT expression levels of autotetraploids peaked in the $12^{\text {th }}$ day, and this was $58.92 \%$ greater than that of diploids (Fig. 4f). The expression of BpProDH and $B p P 5 C D H$ in diploids and autotetraploids showed a trend of decline, and which of autotetraploids were always lower than those of diploids. The minimum of $B p P r o D H$ and $B p P 5 C D H$ expression of autotetraploids appeared in the $12^{\text {th }}$ day, and there were $65.36 \%$ and $35.54 \%$ lower than those of diploids, respectively (Fig. 4g-h).

\section{Discussion}

The natural European aspen (Populus tremula) triploid was discovered by Nilsson-Ehle (1936) and Müntzing (1936) in Sweden. Since then, plant-breeding scientists have given extensive attention to polyploids in forest trees due to the huge growth 

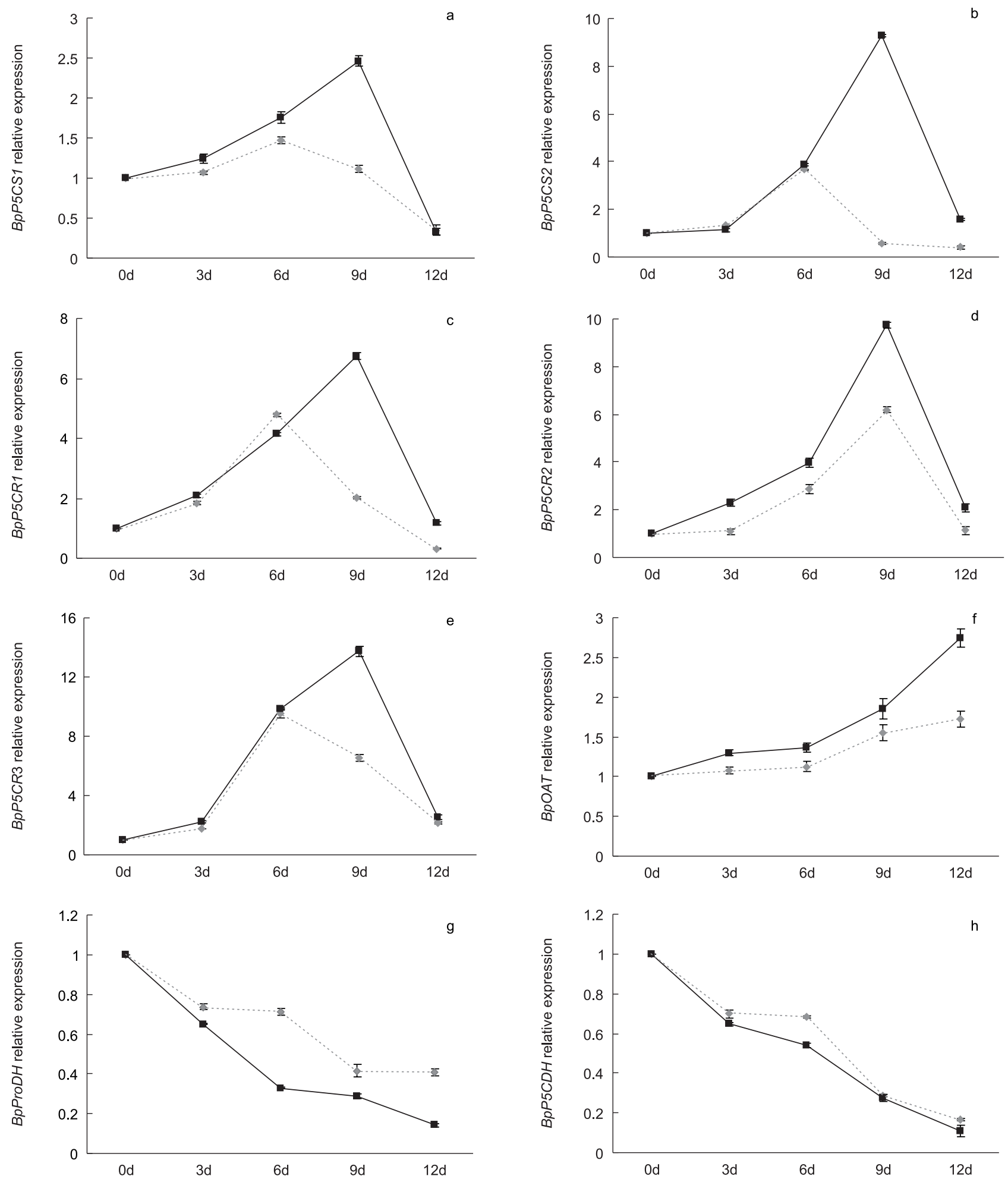

Fig. 4. Proline-associated gene expression of diploids and autotetraploids in $\mathrm{NaHCO}_{3}$ irrigating. (a) BpP5CS1; (b) BpP5CS2; (c) BpP5CR1; (d) BpP5CR2; (e) BpP5CR3; (f) BpOAT; (g) BpProDH; (h) BpP5CDH

of forestry. Research reported that polyploids bear such characteristics as greater growth and higher resistance against biotic and abiotic stress. Triploid birch was more resistant to birch rust (Eifler, 1960).
Pentaploid and hexaploid birch were more tolerant of water deficit than their diploid relatives (Li et al., 1996). Black locust (Robinia pseudoacacia) with tetraploid chromosomes showed higher POD, SOD and 
CAT levels than diploid trees and markedly inhibited peroxide formation (Meng et al., 2008). This present study discovered that the injury index of autotetraploids was lower than that of diploids, and the relative growth of autotetraploids were superior to those of diploids after $\mathrm{NaHCO}_{3}$ stress. These results indicate that autotetraploid trees were more resistant to salt and alkali stress than diploids.

It has been known for decades that proline plays a critical role in regulating osmotic pressure and stabling cellular structure and proline was accumulated in plants under salt and alkali stress (Zhao et al., 2003). Glutamate (Glu) pathway and ornithine (Orn) pathway are proline biosynthetic pathways in plants (Delauney et al., 1993; Chiang \& Dandekar, 1995). In glutamate pathway, glutamate is catalyzed by pyrroline-5-carboxylate synthetase (P5CS) to glutamate-5-semialdehyde (GSA), and glutamate-5-semialdehyde is spontaneously cyclization to pyrroline-5-carboxylate (P5C), and pyrroline-5-carboxylate is catalyzed by pyrroline-5-carboxylate reductase (P5CR) to proline (Giberti et al., 2014; Zhang et al., 2015). In ornithine pathway, ornithine is catalyzed by ornithine- $\delta$-aminotransferase (OAT) to glutamate-5-semialdehyde, and glutamate-5-semialdehyde get into the glutamate pathway to generate proline (Song et al., 2005; You et al., 2012). If proline is over biosynthesized in plants, proline is catalyzed by proline dehydrogenase (ProDH) to pyrroline-5-carboxylate, and pyrroline-5-carboxylate is spontaneously cyclization to glutamate-5-semialdehyde, and glutamate-5-semialdehyde is catalyzed by pyrroline-5-carboxylate dehydrogenase (P5CDH) to glutamate (Senthil-Kumar \& Mysore, 2012; Monteoliva et al., 2014). In the present study, the proline content of autotetraploids was always greater than that of diploids in $\mathrm{NaHCO}_{3}$ irrigating, and the rising period of proline content in autotetraploids was longer than that of diploids for three days. These results suggest an explanation for the resistance to salt and alkali stress in autotetraploid trees. As proline-biosynthesized genes, the variation trend of $B p$ P5CS1, BpP5CS2, BpP5CR1, BpP5CR2 and BpP5CR3 was similar to that of proline content in diploids and autotetraploids, which was a downward after the first rise. Nevertheless, the variation of BpOAT in diploids and autotetraploids showed a rising trend. This phenomenon may be attributed that P5CS and P5CR will be restrained by superfluous proline but nor OAT in plants (Zhao et al., 2008). As proline-degraded genes, the variation of $B p P r o D H$ and $B p P 5 C D H$ in diploids and autotetraploids showed a trend of decline, which may be attributed that $\mathrm{ProDH}$ and $P 5 C D H$ are suppressed by salt and alkali stress in plants (Zhao \& Liu, 1999). In addition, autotetraploids were generally higher expression in BpP5CS1, BpP5CS2, BpP5CR1, BpP5CR2, BpP5CR 3 and BpOAT and were lower expression in $\mathrm{BpProDH}$ and $\mathrm{BpP} 5 \mathrm{CDH}$ compared to diploids, which may be related to the increase of proline content and the resistance to salt and alkali stress in autotetraploid birch.

It is currently inadequate research about resistance in birch polyploids, which is prejudicial to obtain new birch species for forestry. Consequently, it is advisable to make further research about the tolerant to biotic stress and abiotic stress on the basis of the present study, and regard polyploid breeding as a new approach for genetic improvement of birch trees.

\section{Acknowledgement}

This work was financially supported by a grant from the 12th Five Year Science and Technology Program of Jilin Provincial Education Department (2015139) and the Doctoral Scientific Research Foundation of Beihua University.

\section{References}

Ahmed P (1991) Agroforestry: a viable land use of alkali soils. Agroforestry Systems 14: 23-37.

Chiang HH \& Dandekar AM (1995) Regulation of proline accumulation in Arabidopsis thaliana (L.) Heynh during development and in response to desiccation. Plant, Cell \& Environment 18: 12801290.

Delauney AJ, Hu CA, Kishor PB \& Verma DP (1993) Cloning of ornithine delta-aminotransferase cDNA from Vigna aconitifolia by trans-complementation in Escherichia coli and regulation of proline biosynthesis. Journal of Biological Chemistry 268: 18673-18678.

Deng BL, Du WC, Liu CL, Sun WW, Tian S \& Dong HS (2012) Antioxidant response to drought, cold and nutrient stress in two ploidy levels of tobacco plants: low resource requirement confers polytolerance in polyploids? Plant Growth Regulation 66: 37-47.

Eifler I (1960) The individual results of crosses between B. verrucosa and B. pubescens. Silvae Genetica 9: 159-165.

Eriksson G \& Jonsson A (1986) A review of the genetics of Betula. Scandinavian Journal of Forest Research 1: 421-434.

Giberti S, Funck D \& Forlani G (2014) $\Delta^{1}$-pyrroline-5-carboxylate reductase from Arabidopsis thaliana: stimulation or inhibition by chloride ions and feedback regulation by proline depend on whether NADPH or NADH acts as co-substrate. New Phytologist 202: 911-919.

Johnsson H (1953) Development of triploid and diploid Populus tremula during the juvenile period. 
Zeitschrift fur Forstgenetik und Forstpflanzenziichtüng 2: 73-77.

Koski V \& Rousi M (2005) A review of the promises and constraints of breeding silver birch (Betula pendula Roth) in Finland. Forestry 78: 187-198.

Li WL, Berlyn GP \& Ashton PM (1996) Polyploids and their structural and physiological characteristics relative to water deficit in Betula papyrifer. American Journal of Botany 83: 15-20.

Li YY, Yang G, Wei R, Sun YS, Guo YH, Zhang RP \& Liu GF (2013) TabZIP transferred Betula platyphylla generation and salt tolerance analysis. Journal of Nanjing Forestry University (Natural Sciences Edition) 37: 6-12.

Liu L, Huang FL, Luo QX, Pang HY \& Meng FJ (2012) cDNA-AFLP analysis of the response of tetraploid black locust (Robinia pseudoacacia L.) to salt stress. African Journal of Biotechnology 11: 3116-3124.

Löve Á (1944) A new triploid Betula verrucosa. Svensk Botanisk Tidskrift 38: 381-393.

Meng FJ, Wang QY, Wang JZ, Li SY \& Wang JJ (2008) Salt resistance of tetraploid Robinia Pseudoacacia. Chinese Journal of Plant Ecology 32: 654-663.

Monteoliva MI, Rizzi YS, Cecchini NM, Hajirezaei MR \& Alvarez ME (2014) Context of action of proline dehydrogenase (ProDH) in the hypersensitive response of Arabidopsis. BMC Plant Biology 14: 21.

Mu HZ, Liu ZJ, Lin L, Li HY, Jiang J \& Liu GF (2012) Transcriptomic analysis of phenotypic changes in birch (Betula platyphylla) autotetraploids. International Journal of Molecular Sciences 13: 13012 13029.

Müntzing A (1936) The chromosomes of a giant Populus tremula. Hereditas 21: 383-393.

Nilsson-Ehle H (1936) Über eine in der Natur Gefundene Gigasform von Populus tremula. Hereditas 21: 379-382.

Niwa Y \& Sasaki Y (2003) Plant self-defense mechanisms against oxidative injury and protection of the forest by planting trees of triploids and tetraploids. Ecotoxicology and Environmental Safety 55: 70-81.
Senthil-Kumar M \& Mysore KS (2012) Ornithine-delta-aminotransferase and proline dehydrogenase genes play a role in non-host disease resistance by regulating pyrroline-5-carboxylate metabolism-induced hypersensitive response. Plant, Cell \& Environment 35: 1329-1343.

Song SQ, Lei YB \& Tian XR (2005) Proline metabolism and cross-tolerance to salinity and heat stress in germinating wheat seeds. Russian Journal of Plant Physiology 52: 793-800.

Sun ZX, Yang HH, Cui DC, Zhao CZ \& Zhao SP (2002) Analysis of salt resistance on the poplar transferred with salt tolerance gene. Chinese Journal of Biotechnology 18: 481-485.

You J, Hu HH \& Xiong LZ (2012) An ornithine $\delta$-aminotransferase gene OsOAT confers drought and oxidative stress tolerance in rice. Plant Science 197: 59-69.

Zeng FS, Nan N \& Zhan YG (2007) Extraction of total RNA from mature leaves rich in polysaccharides and secondary metabolites of Betula platyphylla Suk. Plant Physiology Communications 43: 913-916.

Zhang DZ, Wang PH \& Zhao HX (1990) Determination of the content of free proline in wheat leaves. Plant Physiology Communications 26: 62-65.

Zhang GC, Zhu WL, Gai JY, Zhu YL \& Yang LF (2015) Enhanced salt tolerance of transgenic vegetable soybeans resulting from overexpression of a novel $\Delta^{1}$-pyrroline-5-carboxylate synthetase gene from Solanum torvum Swartz. Horticulture, Environment, and Biotechnology 56: 94-104.

Zhao FG \& Liu YL (1999) Advances in study on metabolism and regulation of proline in higher plants under stress. Chinese Bulletin of Botany 16: 540-546.

Zhao FY, Guo SL, Wang ZL, Zhao YX \& Zhang H (2003) Recent advances in study on transgenic plants for salt tolerance. Journal of Plant Physiology and Molecular Biology 29: 171-178.

Zhao RX, Zhu HS, Cheng YH \& Dong KH (2008) Research progress on proline and its biosynthesis enzymes in plant. Pratacultural Science 25: 90-97. 